\title{
LVI. On the photography of ripples
}

\author{
J. H. Vincent B.Sc. A.R.C.Sc.
}

To cite this article: J. H. Vincent B.Sc. A.R.C.Sc. (1897) LVI. On the photography of ripples , Philosophical Magazine Series 5, 43:265, 411-417, DOI: 10.1080/14786449708621013

To link to this article: http://dx.doi.org/10.1080/14786449708621013

曲 Published online: 08 May 2009.

Submit your article to this journal

Џll Article views: 7

Q View related articles $₫$ 


\section{[ 411$]$}

LVI. On the Photography of Ripples. By J. H. Vinchert, B.Se., A.R.C.Sc., Assistant Demonstrator in Plysics at the Royal College of Science, London, S.W.*

[Plates I.--1II.]

M ANY of the phenomena described in this paper have been exhibited at public lectures by Mr. (:. V. Boys, using the stroboscopic method, which was first applied to the study of ripples by Lord Rayleigh. I an indebted to $\mathrm{Mr}$. Boys for having recommended to me the work of photographing these effects, and also for many valuable suggestions.

Lord Kolvin defines a ripple as a wave whose length is less than that of the wave which is propagated with the minimum velocity. For ordinary mercury, waves less than $1 \cdot 3$ centim. Iong are ripples. Capillary ripples are those whose length is so small as to render negligible, in the value of the velocity squared, the term due to gravity. These definitions are rendered clearer by reference to Mr. Boys's Logarithmic Wave Chart. The portion of the curve, representing the relation of the velocity and wave-length, to the left of the point of minimum velocity, refers to ripples. The straight-line portion to the left of the chart represents capillary ripples.

In order to obtain ripples it is necessary to use vibrationfrequencies above a certain value. Thus in the case of ordinary clean-looking mercury, with its damp and probably greasy surface, the surface-tension of which may be between 300 and 400 C.G.S. units, a frequency of about 15 per second causes the biggest waves which the above definitions include as ripples; while frequencies of about 200 and upwards give rise to waves whose propagation is practically controlled by surface-tension, and these waves are capillary ripples.

Now the duration of the sensation produced by a luminous impression on the retina lasts for about one eighth of a second; thus we are unable to see ripples on the surface of mercury. The frequencies employed are generally many times the maximum visible frequency. It is not the high velocity of propagation which renders ripples invisible; ripples produced by a disturbance of a frequency of about 200 do not travel very quickly, a foot a second being about the order of magnitude of the velocity on the surfice of ordinary mercury.

* Communicated by the Physical Suciety : read Feb. 26, 1897. 


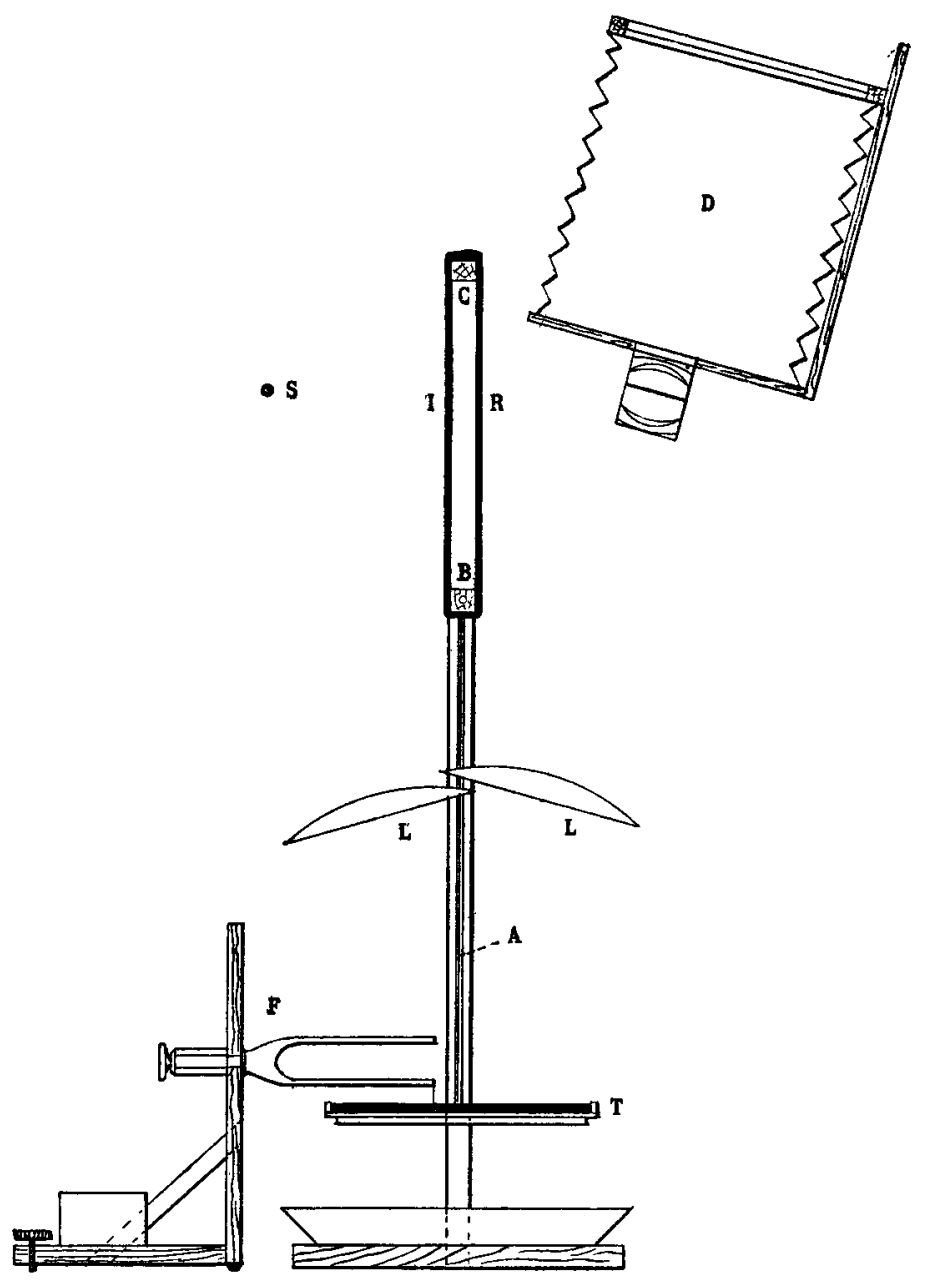

Diagram of Apparatus. $\quad \frac{1}{1} \frac{1}{2}$ nat. size.

T. Mercury trough.

A. Standard of retort-standard.

B. Wooden block through which passes the bent down portion of $\mathrm{A}$.

C. Upper bar of wooden stand.

IR. India-rubber loop.

S. First spark-gap.

LL. Lenses.

D. Camera.

F. Tuning-fork and stand. 


\section{Description of the Apparatus.}

A rectangular wooden trough, about 1 centim. deep and $12 \times 15$ centim. area, contains the mercury upon the surface of which the ripples are produced. This trough rests upon the rectangular base of a retort-stand, the upper portion of whose standard is bent at right angles over the base so as to be parallel with the latter. This horizontal portion is passed through a rectangular block of wood, and the whole is then slung from a gallows-like wooden structure one metre high.

The spark-gap from which the light proceeds to illuminate the mercury surface is placed near the top and to one side of the wooden stand. Light from this gap falls upon a lens to the left of the stand, so that the emergent light is parallel ; after reflexion the light is collected by a second lens similar in all respects to the first, so that an image of the spark would be produced at the primary focus of the second lens; the focal length of these lenses was 44 centim. The camera is placed in such a position as to enable the first achromatic lens of the combination to collect the rays and converge them so as to come to a focus at the aperture in the lens stop. The stop used was the smallest of the set belonging to the camera (F 64). The camera is then focussed upon a fine thread laid on the surface of the mercury.

In addition to the first spark-gap, which was about $\cdot 5$ centim. across, a second gap was used in order to increase the brightness of the spark in the first gap. The second gap was varied from time to time, but was generally 1.5 cention. across. The first gap was shunted by a piece of stout thread soaked in calcium-chloride solution. This prevented small sparks due to induction. The knobs of a Wimshurst machine were connected, one with a terminal of the second spark-gap and the inside coats of a battery of four half-gallon leyden-jars; the other Wimshurst terminal was connected to the outer coats, one side of the first spark-gap, and to earth. A wire joining the other terminals of the two gaps coinpletes the spark arrangements.

\section{Method of Causing the Ripples.}

The ripples are due to the agitation of the surface of the mercury by a style of glass attached to one prong of a vibrating tuning-fork. The fork was in most cases struck with a rubber-shod hammer; but in the last two experiments, in which a strip of cover-glass attached to the fork acts as a linesource, it was found that the irregular large waves caused by the concussion entirely masked the phenomena which it was sought to photograph. In these cases the fork was maintained in synchronous ribration with another similar fork

Phil. Mag. S. 5. Vol. 43. No. 265. June 1897. 2 I 
which was electrically excited. The two forks, placed approximately parallel, are tied together by a piece of thread about two feet long, so that the thread is at right angles to both forks. This thread is then adjusted to an appropriate tension by trial, when the maintained fork causes the other to vibrate for any length of time. This simple device, which has been used in the Royal College of Science laboratory for some time, is due to Mr. W. Watson.

\section{Deseription of Photographs.}

The plates used were of various kinds, and any of the wellknown plates gave good results. Some of the negatives were intensified previous to printing. The figures are about $\frac{1}{2}$ natural size. My best thanks are due to Mr. R. Chapman, who has assisted me throughout with great zeal.

Photographs showing a series of circular waves set up by a single style attached to a fork of known frequency were taken with a view to quantitative measurements of surfacetension, wave-length, and velocity. A bar of wood was so placed that two needle-points which it carried nearly touched the surface of the mercury. These points are a known distance apart, and by measuring the negatives we may find the scale of reduction of lengths along this line. The particulars of the motion are obtained from the equations

$$
v^{2}=n^{2} \lambda^{2}=\frac{g \lambda}{2 \pi}+\frac{2 \pi \mathrm{T}}{\lambda \rho} ;
$$

from which we find, in a particular experiment,

$$
\begin{aligned}
n & =180 \text { per second, } \\
\lambda & =\cdot 165 \text { centim., } \\
v & =29 \cdot 7 \text { centim. a second, } \\
\mathrm{T} & =306 \text { dynas per linear centim. }
\end{aligned}
$$

This low value of the surface-tension was obtained from mercury which had stood in the apparatus for some days. The values obtained in a similar way previously were 420 , 421,365 . The tension falls as the mercury gets more contaminated. The value of the surface-tension of pure dry mercury is usually quoted at 540 .

Fig. 1. Two styles are attached to the same prong of a fork, the frequency of which is 120 . One centre is untortunately hidden by the fork. The approximately straight dark lines which are seen to radiate from the region between the centres of oscillation are lines of minimum disturbance; they are hyperbolas of which the centres of disturbance are the foci. This photograph illustrates the interference phenomena in Optics produced by Young's or Fresnel's methods.

Fig. 2. The frequency of the fork is 256 . Both styles are 
attached to the same prong. The photograph shows two series of interference-curves, one a family of hyperboliss analogous to those shown in fig. 1, and the other a family of ellipses.

The hyperbolas are the radiating light lines seen on the side of the photograph remote from the fork. They are fixed in position, the little dark facets moving along between pairs of hyperbolas.

The light oval curves in the region between the centres of disturbance are ellipses, since they are the loci of points of intersection of the two series of eircles whose radii grow uniformly, and at the same rate with time. Their method of production here is similar to a well-known geometrical construction for ellipses. Unlike the system of hyperbolas, these ellipses are not at rest. They travel outwards in such a way that any ellipse occupies a position which was filled previously by its predecessor a whole period before. That semiaxis of any ellipse which passes through a centre of dis. turbance grows with the same velocity as that with which the ripples are propagated. The other semiaxis grows with a velocity which is infinite at the commencement, but which gradually decreases to the same uniform velocity of growth as that of the first. The law of decrease of velocity is the same as the law of decrease of the lengths of whole-period elements of a linear wave with respect to a point.

In order to render these ellipses stationary it would be necessary to change one of the sources into a sink to which the circular waves converge. This could be experimentally realized with ripples by causing a circular arc and a style to be agitated by the same prong of a fork, when the effects would be analogous to M. Meslin's experiment in Optics.

Fig. 3. Frequency 256.

This photograph is very similar to fig. 1 ; but in addition to showing interference phenomena like those of Fresnel and Young, it also illustrates interference effects in which the direction of propagation of light is parallel to the line joining the point-sources. Thus in the photograph, if we consider the disturbance anywhere on a right line drawn perpendicular to a line joining the two point-sources produced, we see that the places of no disturbance are symmetrical about the line joining the sources. They are points on the system of hyperbolas already mentioned.

In M. Meslin's method of producing interference-fringes the screen is placed between the two point-centres, one a source and the other a sink. 'The bands are circular, and are sections of ellipsoids of revolution, and not of hyperboloids, such as the fringes in the photograph would become if the whole picture were rotated about the line joining the point2 I 2 
sources. These fringes are not seen on the screen in M. Meslin's experiment with the split lens, even when the screen is placed beyond the second focus, because the pencils do not there overlap. It seems that modifications of M. Meslin's experiment could be devised so as to enable complete circular fringes to be seen, and also to render the sections of hyperboloids visible. For example, it appears probable that if a circular portion of a convex lens were cut out and the central portion moved towards the original pointsource, the sections of hyperboloids of revolution would be visible on a screen placed beyond the second focus.

Fig. 4. The two sets of ripples are produced by a fork of frequency 128 and another of frequency about 112. These two forks then produce 16 beats a second. The curved light lines represent places of minimum disturbance at the instant when the spark occurred. These lines are not stationary as in No. 3, but rotate towards their convexities. The centre of disturbance from which they move is the one of higher frequency. If we consider a point anywhere on the surface of the mercury, beats occur at that point with the same frequency as the passage of these lines of ninimum disturbance takes place over the point. Thus, 16 of these lines cross any point per second.

Fig. 5. This shows ripples produced by two forks, the higher of which has a frequency four times as great as the lower, the frequency of which is 128 . If we neglect the effect of gravity,

$$
v^{2}=n^{2} \lambda^{2}=\frac{2 \pi \mathrm{T}}{\lambda \rho}
$$

from which it follows that the wave-length of the ripples from the higher fork should be half that dne to the lower. This relation is approximately true for these ripples.

Fig. 6. Frequency 180.

This photograph show's a point-source and a reflecting-line, the latter is a side of a triangular piece of microscope coverglass, which is kept in position by a small splinter of wood. The interference-lines which are shown are due to the mutual action of the primary and the reflected waves. The phenomena exhibited are analogous to Lloyd's single-mirror fringes in Optics.

Faint signs of diffraction invading the geometrical shadow of the obstacle can be seen. The region of shadow is covered by faint lines parallel to the nearest side of the triangle acting as a line-source. The wave-length is the same as that of the primary waves, and the effect is due to forced vibrations.

Fig. 7. This photograph illustrates reflexion and forced 
vibrations. The light curved lines in the region between the source and the nearest side of the triangle are similar to those between the two sources in fig. 2. They are due to the interference between the source and its virtual image.

Fig. 8. Frequency 256.

Here we have a shallow circular reflector with the source placed approximately at the principal focus. The reflected waves are circles of large radii ; the very slight ontward curvature at the ends shows that even when the reflector has an are of about $60^{\circ}$ the effect of spherical aberration is small. Since the reflected waves come from a virtual point-source, we have, as interference-lines, a series of confocal ellipses and confocal hyperbolas; the latter are fixed, but the former travel away from the line joining the source and its image. If the reflected waves had been rectilinear, both these sets of curves would have become parabolas.

Fig. 9. Frequency $256^{\circ}$.

The centre of disturbance here coincides very closely with the principal focus of the central portion of the semicircular reflector. The reflected ripples are straight lines in the middle, but are bent outwards from the reflector towards the ends. This illustrates spherical aberration.

Fig. 10. Frequency 256.

The obstacle is a small round cover-slip floating on the mercury. The ripple-shadow is slightly encroached upon by the waves bending round the edge of the obstacle. One side of the disk acts as a convex circular mirror, and the interference-fringes are due to the mutual action of the source and its virtual image situated within the cireumference of the disk.

Fig. 11. The frequency in this and fig. 12 is 120.

Here straight-line waves are originated by the agitation of a slip of cover-glass, one side of which dips into the mereury. The waves are reflected from the shallow circular mirror, and converge to the principal focus. Two series of parabolic interference-fringes are shown. They are confocal, and have their concavities directed towards the source and reflector respectively.

Fig. 12. Similarly excited waves are reflected at an angle of about $45^{\circ}$ from a straight edge. The long black mark running from one end of the dipping edge to the corner of the print is due to a depression in the surface caused by a floating needle, put there to screen off the circular waves coming from the end of the strip of glass.

Diffraction is well shown in this photograph. 
Phil. Mag. S. 5. Vol. 43. Pl. I.

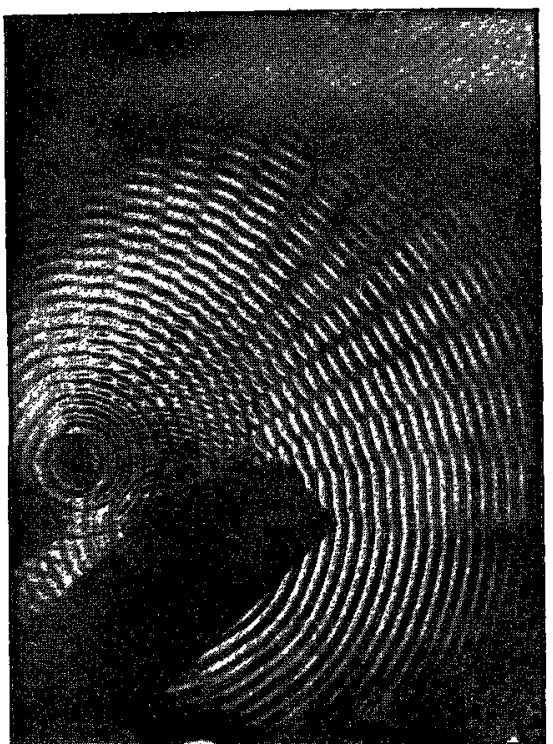

Fig. I,

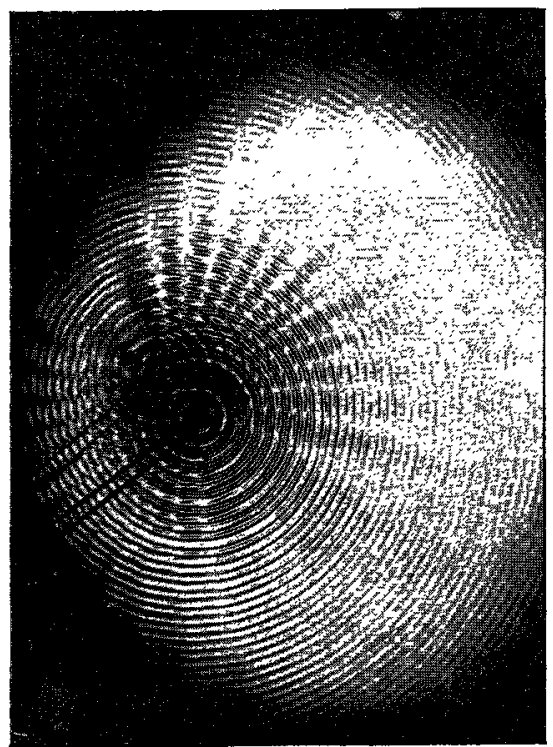

Fig. 3.

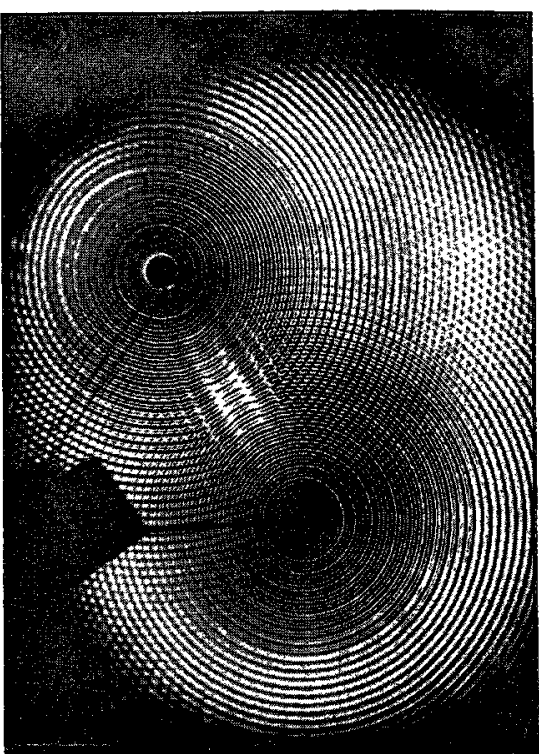

Fig. 2.

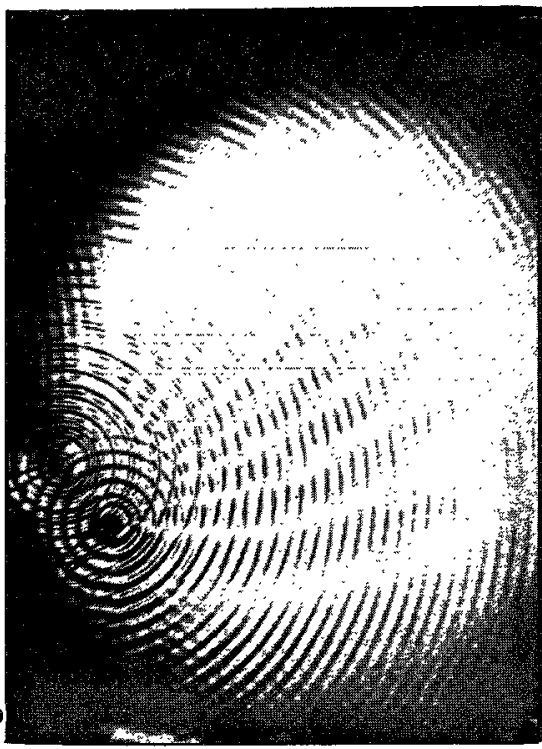

Fig. 4 
Phil. Mag. S. 5. Vol. 43. Pl. II.

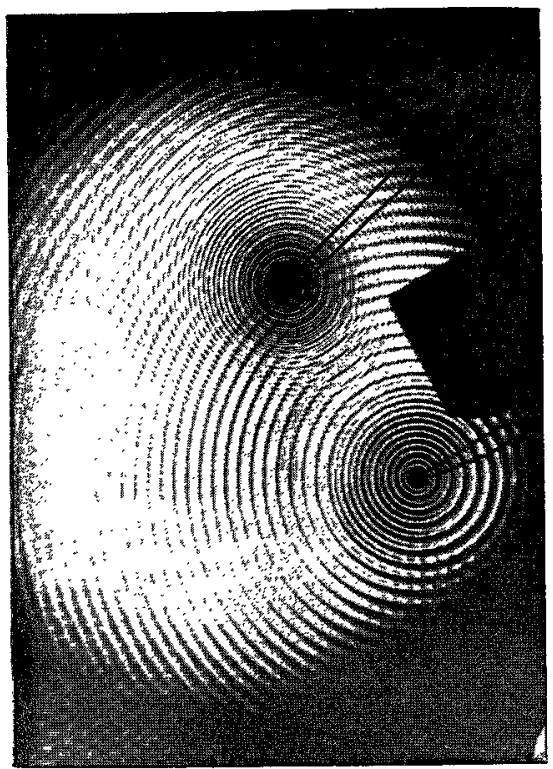

Fig. 5.

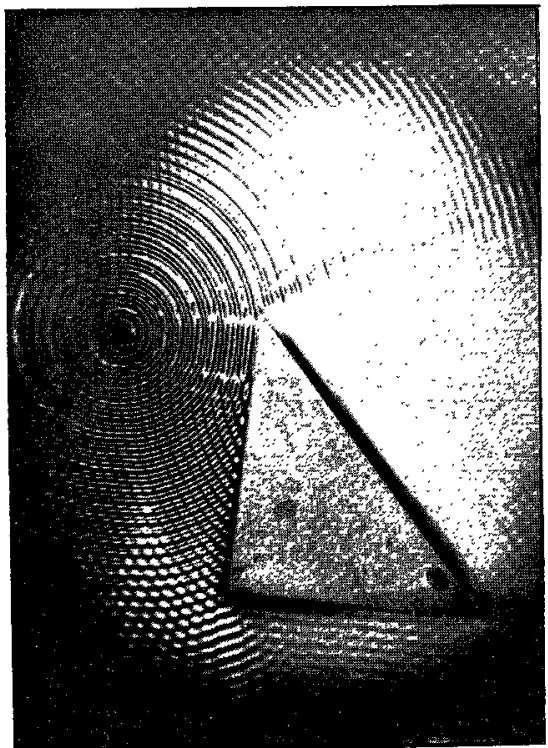

Fig. 7.

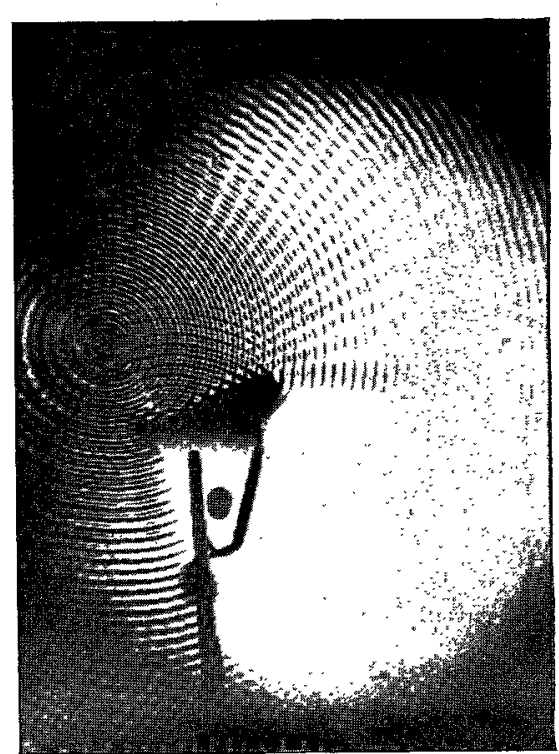

Fig. 6.

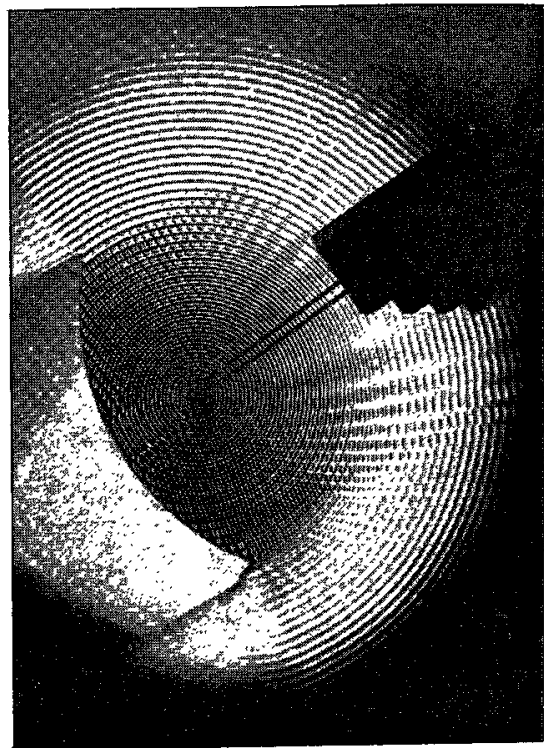

Fig. 8. 
Phil. Mag. S. 5. Vol. 43. Pl. III.

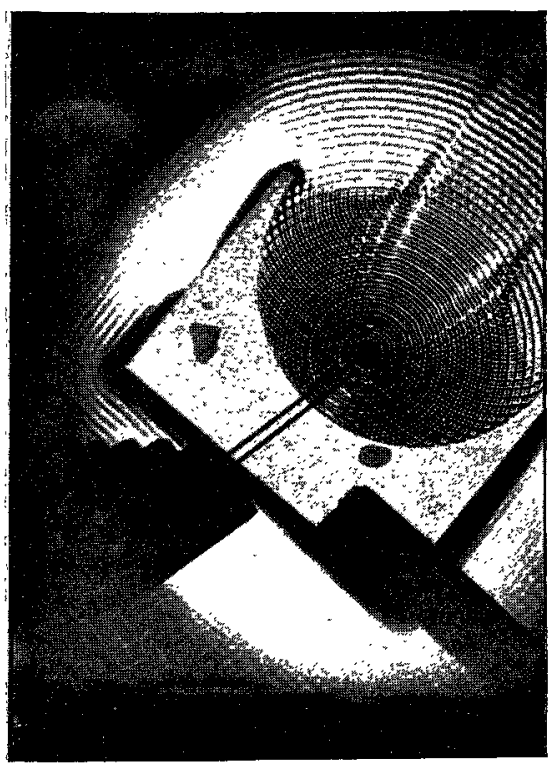

I ig. 9.

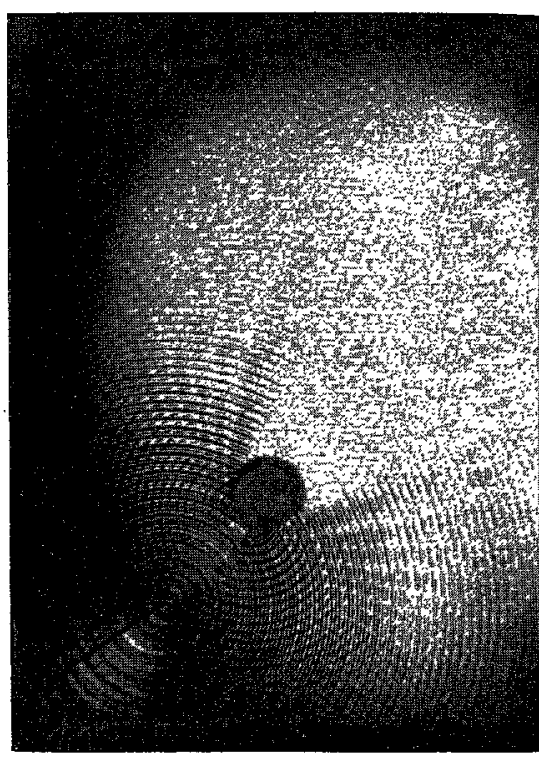

Fig. ro.

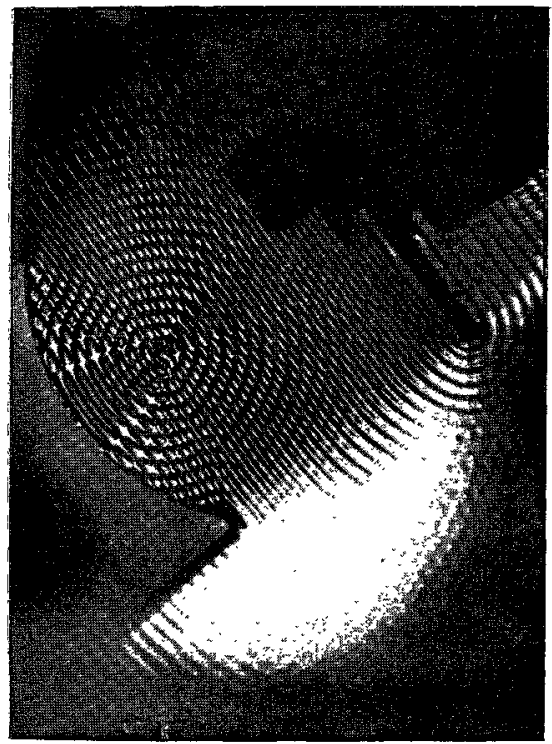

Fig. $\mathbf{r}$.

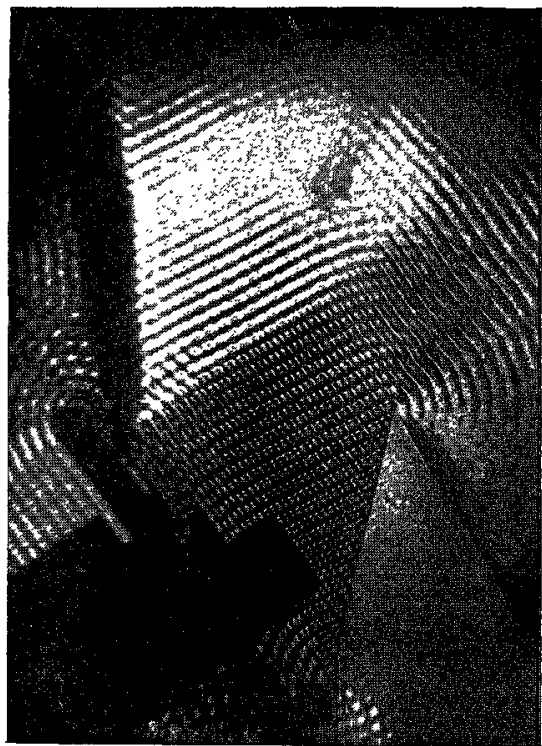

Fig. I2 